\title{
ABSOLUTELY STABLE GAMES
}

\author{
ROBERT JAMES WEBER
}

\begin{abstract}
Absolutely stable games, in which every monotone chain of domination reduces to direct domination, are explicitly characterized. Simple games, and $n$-person games in which all minimal-vital coalitions contain at least $n-1$ players, are seen to satisfy the characterization.
\end{abstract}

Harsanyi [1, pp. 1477-1479] has considered games for which all von Neumann-Morgenstern solutions exhibit a strong form of internal stability. The class of such games includes all absolutely stable games, which Harsanyi defined by a property of chains of domination. It is our purpose to give an explicit characterization of these absolutely stable games.

Let $v$ be a $(0,1)$-normalized monotonic game, with player set $N$. A coalition $T$ is minimal-vital if $v(T)>0$ and if for every $S \varsubsetneqq T, v(S)=0$. A monotone chain is a sequence of imputations and coalitions $\left(x_{0}, S_{1}\right.$, $\left.x_{1}, \ldots, S_{m}, x_{m}\right)$ satisfying

(1) $x_{k-1} \operatorname{dom}\left(S_{k}\right) x_{k}$, and

(2) $\left(x_{0}\right)_{i}>\left(x_{k}\right)_{i}$ for all $i \in S_{k}$,

for $1 \leqslant k \leqslant m$. The game is absolutely stable if for every monotone chain $\left(x_{0}\right.$, $\left.S_{1}, x_{1}, \ldots, S_{m}, x_{m}\right)$, it follows that $x_{0}$ dominates $x_{m}$.

THEOREM. A game $v$ is absolutely stable if and only if

(a) for every minimal-vital coalition $T$, if $N \supsetneqq S \supseteq T$ then $v(S)=v(T)$, and

(b) for every minimal-vital coalition $T$ with $v(T)<1$, every other coalition $S$ with $v(S)>0$ satisfies either $S \supseteq T$ or $S \supseteq N-T$.

Proof. Sufficiency. Assume that the conditions are satisfied, and consider a monotone chain $\left(x_{0}, S_{1}, x_{1}, \ldots, S_{m}, x_{m}\right)$. We will show that $x_{0} \operatorname{dom}\left(S_{m}\right) x_{m}$. By (a), all the coalitions of the chain can be assumed to be minimal-vital. By (2), $\left(x_{0}\right)_{i}>\left(x_{m}\right)_{i}$ for all $i \in S_{m}$. To begin, notice that $x_{m-1}\left(S_{m}\right) \leqslant v\left(S_{m}\right)$, where for notational convenience we generally write $x(S)=\Sigma_{i \in S}(x)_{i}$. Proceeding by induction, we assume $x_{k}\left(S_{m}\right) \leqslant v\left(S_{m}\right)$ and consider $x_{k-1}$. If $v\left(S_{m}\right)=1$, then

$$
x_{k-1}\left(S_{m}\right) \leqslant x_{k-1}(N)=1=v\left(S_{m}\right) .
$$

Otherwise (b) applies to $S_{m}$, and either (i) $S_{k} \supseteq S_{m}$, or (ii) $S_{k} \supseteq N-S_{m}$.

If (i) applies, then since $S_{k}$ is minimal-vital, it follows that $S_{k}=S_{m}$ and therefore

Received by the editors January 30, 1975.

AMS (MOS) subject classifications (1970). Primary 90D12.

Key words and phrases. $n$-person games, von Neumann-Morgenstern solutions. 


$$
x_{k-1}\left(S_{m}\right)=x_{k-1}\left(S_{k}\right) \leqslant v\left(S_{k}\right)=v\left(S_{m}\right) .
$$

On the other hand, if (ii) applies, then $x_{k-1}\left(N-S_{m}\right)>x_{k}\left(N-S_{m}\right)$, and therefore

$$
x_{k-1}\left(S_{m}\right)<x_{k}\left(S_{m}\right) \leqslant v\left(S_{m}\right),
$$

the last inequality following from the induction hypothesis. Thus in any case it eventually follows that $x_{0}\left(S_{m}\right) \leqslant v\left(S_{m}\right)$, and therefore $x_{0} \operatorname{dom}\left(S_{m}\right) x_{m}$.

Necessity. Assume that $T$ is minimal-vital with $v(T)<1$, and consider any $S$ for which $v(S)>0, S \nsupseteq T$, and $S \nsupseteq N-T$. Then $R=N-(S \cup T)$ $\neq \varnothing$ and $T-S \neq \varnothing$. We write $n, r, s, t$ for the respective cardinalities of $N$, $R, S, T$. Let

$$
\begin{aligned}
& x_{i}=\left\{\begin{array}{l}
(1-(2 s+r) \varepsilon) /(t-s) \text { if } i \in T-S, \\
2 \varepsilon \text { if } i \in S, \\
\varepsilon \text { if } i \in R,
\end{array}\right. \\
& y_{i}=\left\{\begin{array}{l}
\varepsilon \text { if } i \in S \cup T, \\
(1-(n-r) \varepsilon) / r \text { if } i \in R,
\end{array}\right. \\
& z_{i}=\left\{\begin{array}{l}
0 \text { if } i \in T, \\
1 /(n-t)
\end{array} \text { if } i \in N-T .\right.
\end{aligned}
$$

Then for sufficiently small $\varepsilon>0, x \operatorname{dom}(S) y \operatorname{dom}(T) z$. However, $x$ does not dominate $z$, since $x(T)>v(T)$. Hence $v$ is absolutely stable only if for every minimal-vital $T$, either $v(T)=1$, or every $S$ with $v(S)>0$ satisfies either $S \supseteq T$ or $S \supseteq N-T$. This establishes the necessity of (b).

Next, assume that $T$ is minimal-vital with $v(T)<1$, and assume that (a) fails. Then there is a minimal nonempty coalition $S$ for which $S \cap T=\varnothing$, $S \cup T \neq N$, and $v(S \cup T)>v(T)$. Select any $k \in R=N-(S \cup T)$, and let

$$
\begin{aligned}
& x_{i}=\left\{\begin{array}{l}
(v(T)+\varepsilon) / t \quad \text { if } i \in T, \\
\varepsilon \text { if } i \in S, \\
1-v(T)-(s+1) \varepsilon \quad \text { if } i=k, \\
0 \text { if } i \in R, i \neq k,
\end{array}\right. \\
& y_{i}= \begin{cases}\varepsilon & \text { if } i \in T, \\
0 & \text { if } i \in S, \\
(1-t \varepsilon) \text { if } i \in R,\end{cases} \\
& z_{i}= \begin{cases}0 & \text { if } i \in T, \\
1 /(n-t) & \text { if } i \in N-T .\end{cases}
\end{aligned}
$$

Then for sufficiently small $\varepsilon>0, x \operatorname{dom}(S \cup T) y \operatorname{dom}(T) z$. However, $x$ $\operatorname{dom}(W) z$ for all small $\varepsilon$ only if $W=T \cup\{k\}$ and $v(W)=1$. In this case, let

$$
x_{i}=\left\{\begin{array}{l}
(v(T)+\varepsilon) / t \quad \text { if } i \in T \\
1-v(T)-\varepsilon \quad \text { if } i=k \\
0 \quad \text { if } i \in N-W
\end{array}\right.
$$




$$
\begin{aligned}
& y_{i}= \begin{cases}\varepsilon & \text { if } i \in T, \\
0 & \text { if } i=k, \\
(1-t \varepsilon) /(n-t-1) & \text { if } i \in N-W,\end{cases} \\
& z_{i}= \begin{cases}0 & \text { if } i \neq k, \\
1 & \text { if } i=k .\end{cases}
\end{aligned}
$$

Then for sufficiently small $\varepsilon>0, x \operatorname{dom}(W) y \operatorname{dom}(T) z$, but $x$ does not dominate $z$. Hence $v$ is absolutely stable only if (a) holds. This completes the proof of the theorem.

REMARK. All simple games (games in which each $v(S)$ is either 0 or 1) satisfy (a) because of monotonicity, and trivially satisfy (b). Games in which all minimal-vital coalitions have at least $n-1$ players trivially satisfy (a), and are easily seen to satisfy (b). Games of these types were first shown to be absolutely stable in [1]. As an example of a game in neither class, $v$ defined by

$$
\begin{aligned}
v(\{1,2\}) & =v(\{1,2,3\})=v(\{1,2,4\})=1 / 2, \\
v(\{1,3,4\}) & =1 / 4, \quad v(\{2,3,4\})=4 / 5, \quad v(\{1,2,3,4\})=1,
\end{aligned}
$$

and

$$
v(S)=0 \text { otherwise, }
$$

is an absolutely stable four-person game.

\section{BIBLIOGRAPHY}

1. John C. Harsanyi, An equilibrium-point interpretation of stable sets and a proposed alternative definition, Management Sci. 20(1974), 1472-1495.

Department of Mathematics, University of Connecticut, Storrs, Connecticut 06268 